requested from both sites in the trust. The standards to compare were identified and information collected in a proforma. This information was later summarised using Excel spreadsheet.

Results 31 case-notes where skeletal survey (SS) was performed over these two periods were reviewed, of these 17 cases were suspected physical abuse, 12 were for unexpected child death/SUDI and 2 were for genetic reasons.

- 17/31 skeletal surveys studied were for presumed NAI, -4/17 before (2007-08); and 13/17 (2009-10) after 2008 guidelines - 2/14 for genetic conditions, $12 / 14$ for child death or SUDI

- Of 17/31 with presumed NAI, presentation included Bruises 10/17 - Fracture 3/17 - Scalp swelling 4/17 (1 had bruising and scalp swelling) - Occult - suspected shaken baby syndrome

- Communication between paediatrician and carers poorly documented - Concerns 1/3rd (pre) and 2/3rd cases (post) Explanation of imaging $0 / 3$ (pre) and $1-3 / 12$ (post) - Consent for imaging 0 (pre) and 0 (post)

- Communication between Paediatricians and Radiologists poorly documented, only 2-3/17 cases

- Good performance with respect to - Timing ( $<1$ day), 3/4 (pre) and 8/13 (post) - Completeness of Skeletal Survey 4/4 (pre) and 12/13 (post) - Verbal report ( $<1$ day) $4 / 4$ (pre) and $12 / 13$ (post) - Final report (<1 day) $2 / 4$ (pre) and $11 / 13$ (post)

- Report, scope for improvement in -Age of injury $2 / 2$ (pre) and $1 / 3$ (post) - Bone density $3 / 4$ pre) and $2 / 13$ (post) - Differential diagnosis $1 / 2$ (pre) and 4/7 (post)

- Additional information from skeletal survey $2 / 17,(\sim 12 \%)$

Summary The study revealed good performance in completing and reporting skeletal survey but documentation of concerns, explaining pathway, sharing concerns with radiologist and some aspects of reporting were not consistent.

\section{G228(P) CHALLENGES OF REFERRING CHILDREN TO SOCIAL CARE FOR REFUGEE COMMUNITY ORGANISATIONS}

doi:10.1136/archdischild-2013-304107.240

'J Thomas, ${ }^{2} \mathrm{C}$ Hands. ${ }^{1}$ Paediatrics, Newham University Hospital, London, UK; ${ }^{2}$ Neonates, Imperial College Healthcare, London, UK

Aims To describe the reasons why refugee community organisations make safeguarding referrals to children's social care. To describe the actions taken by children's social care after such referrals have been made and their effects.

Methods All safeguarding referrals made by one London refugee community organisation between December 2002 and December 2012 were reviewed. The categories of abuse under which each referral was made, other relevant factors and the outcomes of the referrals were recorded.

Results

Abstract G228(P) Table 1

\begin{tabular}{ll}
\hline Category & \\
\hline Neglect & 4 \\
Neglect + physical abuse & 4 \\
Neglect + physical abuse + emotional abuse & 3 \\
Neglect + emotional abuse & 2 \\
Physical abuse & 5 \\
Physical abuse + emotional abuse & 4 \\
Emotional abuse & 3 \\
Sexual abuse & 1 \\
No category stated & 1 \\
\hline
\end{tabular}

\section{Abstract G228(P) Table 2}

\begin{tabular}{ll}
\hline Outcome & \\
\hline Assessment done & 21 \\
Result & 18 \\
No action & 4 \\
Child in need & 4 \\
Child protection plan & 1 \\
Other intervention & \\
Effect on relationship with organisation & 17 \\
Continued as before & 5 \\
Continued after a break & 5 \\
Permanently broken & \\
\hline
\end{tabular}

Conclusions Refugee community organisations frequently make referrals to children's social care. The referral and social care assessment can lead to the breakdown of the relationship between the organisation and the family concerned, leading to a loss of support for the child. Enhanced forums for discussing the cases of vulnerable children with social care outside of making a formal referral are needed to improve the coordination of services.

\section{British Society for Paediatric and Adolescent Rheumatology}

\section{G229 A, B, C, DON'T EVER FORGET THE JOINTS! - A YORKSHIRE PAEDIATRIC RHEUMATOLOGY NETWORK AUDIT}

doi:10.1136/archdischild-2013-304107.241

'WM Abusrewil, 'A Whiteley, ${ }^{2} \mathrm{~A}$ McMahon, ${ }^{2} \mathrm{M}$ Al-Obaidi. 'Paediatrics, Leeds Teaching Hospitals Trust, Leeds, UK; '2Paediatric Rheumatology, Sheffield Children's Hospital, Sheffield, UK

Aims To assess if the musculoskeletal (MSK) examination was being performed, in eight paediatric centres across Yorkshire, when the clinical situation would suggest it was warranted, using the pGALS examination tool ${ }^{1}$

Methods 397 case notes were randomly collected and reviewed retrospectively for patients who had presented to hospital between August-November 2012. Each centre assessed approximately 50 sets of patient's notes

The admission notes were reviewed to find out whether a MSK examination had been documented by the doctors on the initial assessment and then the first and second reviews, if there were triggers or red flags for the examination. ${ }^{1}$

Evidence of documentation of the systemic clinical examinations performed and pertinent clinical variables, including times of admission and the grade of the doctors reviewing patients, were noted.

The information was collected on excel spreadsheets at source hospitals and collated by the audit team to investigate the trends across Yorkshire.

Results $35 \%$ of the 397 admissions had a trigger for a MSK examination.

$26 \%$ of the 397 admissions had a red flag for a MSK examination.

Not a single patient who needed a MSK examination on initial assessment or first review had a full MSK examination documented. In comparison $80 \%$ of patients routinely had a respiratory and cardiovascular examinations documented on initial assessment.

Only 1 out of 105 patients who had a red flag for a MSK examination had a complete examination documented.

Conclusions In 2004 the musculoskeletal examination was shown to be poorly documented. ${ }^{2}$ This audit shows that the musculoskeletal 
examination is still rarely being documented across Yorkshire, across disciplines, by doctors at all levels, even when there are clear triggers and red flags for the examination.

We propose a change in the admission documentation regionally to include a specific MSK section for acute admissions.

We plan educational events in the region to improve knowledge of MSK examination

We plan to re-audit in the region, following the introduction of this intervention.

We propose a national audit should be performed. then more educational measures, directed at all levels of doctors should be driven forward, on a national level, to ensure that this picture improves.

\section{REFERENCES}

1. pGALS- A screening examination of the musculoskeletal system in school aged children, Foster HE, Jan dial S, 'Hands On', Reports on the Rheumatic Diseases Series 5, Number 15, June 2008.

2. Rheumatology (Oxford). 2004 Aug; 43(8):1045-9. EPub 2004 Jun 8. More 'cries from the joints': assessment of the musculoskeletal system is poorly documented in routine paediatric clerking. Goff I, Bateman B, Myers A, Foster H

\section{G230 THE USE OF COMPOSITE SCORES FOR THE ASSESSMENT OF JUVENILE IDIOPATHIC ARTHRITIS (JIA) IN A ROUTINE OUTPATIENT CLINICAL SETTING}

doi:10.1136/archdischild-2013-304107.242

C Xie, P Davis, J McDonagh, K Bailey, C Ryder, P Davies, T Southwood. Rheumatology, Birmingham Children's Hospital, Birmingham, UK

Several JIA composite scores have been validated for use in clinical research studies, but the practicality of their use in the routine clinical setting is unclear. Our aim was to determine the completion rate of the 6 item composite disease activity score, the American College of Rheumatology core outcome variables (ACR COV), and to calculate the 4 item composite Juvenile Arthritis Disease Activity Score (JADAS) in a tertiary rheumatology unit outpatient clinic setting.

Methods In this single centre clinical service evaluation, a retrospective case note review of 105 consecutive JIA patients attending outpatient follow up clinics in 2011-12 was undertaken. The completion rate of a standardised ACR COV proforma (present in all case notes) was determined, and JADAS was calculated from additional clinical data. Pearson's correlation and logistic regression were used to assess the impact of individual items on changes in JADAS. Results 105 children with JIA had 193 clinic visits but complete ACR COV data were found in only $68 / 193$ records (35\%). Of the 6 items comprising the ACR COV, the ESR accounted for the majority of missing data. Sufficient data was available to calculate JADAS scores in 22 children with two consecutive outpatient visits. The table indicates that changes in JADAS were most dependent on the physician's global assessment, and least dependent on ESR (Pearson correlation). Stepwise regression showed that the physician's global assessment alone predicted $87.5 \%$ of JADAS change and the ESR contributed an additional $3.3 \%$

\section{Abstract G230 Table 1}

\begin{tabular}{|c|c|c|c|}
\hline \multicolumn{2}{|c|}{ Pearson correlation } & \multicolumn{2}{|c|}{ Stepwise regression } \\
\hline & Change in JADAS & & Change in JADAS \\
\hline $\begin{array}{l}\text { Physician global } \\
\text { assessment }\end{array}$ & 0.93 & $\begin{array}{l}\text { Physician global } \\
\text { assessment }\end{array}$ & 87.5 \\
\hline Active joints & 0.84 & ESR & 90.8 \\
\hline $\begin{array}{l}\text { Patient global } \\
\text { assessment }\end{array}$ & 0.73 & $\begin{array}{l}\text { Patient global } \\
\text { assessment }\end{array}$ & 97.1 \\
\hline ESR & 0.64 & Active joints & 98.8 \\
\hline
\end{tabular}

Conclusion In a routine clinical setting, frequent missing data reduced the potential clinical utility of the ACR COV and JADAS composite scores. We speculate that a composite clinical score which does not rely on recording the ESR may improve completion rates without diminishing clinical utility.

\section{G231 MORTALITY PATTERNS IN CHILDREN WITH JUVENILE DERMATOMYOSITIS: TWO DECADES OF EXPERIENCE FROM A SINGLE TERTIARY CARE CENTRE}

doi:10.1136/archdischild-2013-304107.243

'S Singh, 'D Suri, 'R Aulakh, 'A Gupta, 'A Rawat, ${ }^{2} \mathrm{R}$ Manojkumar. 'Department of Paediatrics, Post Graduate Institute of Medical Education and Research, Chandigarh, India; ${ }^{2}$ Department of Cardiology, Post Graduate Institute of Medical Education and Research, Chandigarh, India

Introduction Juvenile dermatomyositis (JDM), is a chronic inflammatory disease characterised by proximal muscle weakness and pathognomonic rash. Although there have been significant advances in management of JDM, the condition still has significant morbidity and mortality associated with it. Complications like breakthrough infections, gastrointestinal tract vasculitis, interstitial pneumonitis and myocarditis pose significant challenges to treating physicians. We report our experience of mortality patterns in this condition over the last 2 decades. There is paucity of literature on this aspect of JDM.

Patients and methods Case files of 72 patients diagnosed to have JDM at a single centre during the period January 1993-November 2012 were reviewed. The total follow-up amounts to 256 patient years. The diagnosis of JDM was based on Bohan and Peter criteria. Standard treatment protocols (based on glucocorticoids and methotrexate) were used in management and the senior author was involved in the care of all these children. We recorded 7 deaths amongst these patients over the last 2 decades.

Results Amongst the 7 children who died, there were 5 boys and 2 girls. The mean age at diagnosis was 10.2 years (range $7-12$ years) and duration of treatment ranged from 1 month to 9 years. The causes of death included: upper gastrointestinal vasculitis (3 patients) - of the latter, 1 had perforation peritonitis and 1 had massive upper gastrointestinal bleeding leading to sudden death even before medical aid could be provided; interstitial lung disease with secondary infection (2 patients); bronchiolitis obliterans with organising pneumonia and pneumothorax (1 patient); progressive muscle disease with cardiomyopathy refractory to immunosuppesive therapy (1 patient) - this child died after 9 years of follow-up. Intercurrent and breakthrough infections were recorded amongst 5 of these 7 children.

Conclusion JDM is associated with significant mortality $-13.8 \%$ at our centre over the last 2 decades. Gastrointestinal and pulmonary involvement remain the most common causes of death in this condition. Infections are also an important contributory cause. In our experience, delays in diagnosis and referral contribute significantly to mortality in this condition.

\section{G232 USE OF INFLIXIMAB IN CHILDREN WITH REFRACTORY KAWASAKI DISEASE}

doi:10.1136/archdischild-2013-304107.244

'S Singh, 'D Suri, ${ }^{2 R}$ Manojkumar, ${ }^{2} \mathrm{~N}$ Narula, 'A Gupta, 'A Rawat. 'Department of Paediatrics, Post Graduate Institute of Medical Education and Research, Chandigarh, India; 'Department of Cardiology, Post Graduate Institute of Medical Education and Research, Chandigarh, India

Introduction The aetiology of Kawasaki diasese (KD) is still an enigma. It is known that cytokines have a major role to play in the pathogenesis of this condition. There is evidence of TNF- $\alpha$ 